\title{
FOREWORD
}

\section{Surface nanopatterns induced by ion-beam sputtering}

\author{
Guest Editors \\ Rodolfo Cuerno \\ Departamento de \\ Matemáticas, Universidad \\ Carlos III de Madrid, Spain \\ Luis Vázquez \\ Instituto de Ciencia de \\ Materiales de Madrid, \\ Consejo Superior de \\ Investigaciones Científicas, \\ Spain
}

\section{Raúl Gago}

Instituto de Ciencia de

Materiales de Madrid,

Consejo Superior de

Investigaciones Científicas,

Spain

\author{
Mario Castro \\ Escuela Técnica Superior de \\ Ingeniería (ICAI), \\ Universidad Pontificia \\ Comillas de Madrid, Spain
}

Reports on the production of submicron and nanometric patterns on the surfaces of solid targets eroded by ion irradiation (sputtering) at low and medium energies date back to at least the 1960s with the pioneering works by Cunningham et al [1] and Navez et al [2]. However, only with the advent of high resolution tools for surface and interface characterization was the high potential of this procedure recognized as a method for efficien production of well ordered surface patterns. Such morphologies are made up of periodic arrangements of nanometric sized features, that may show properties of high technological interest. In particular, while periodic ripple morphologies had been well documented since their early observations $[1,2]$, to some extent the observation of ordered arrays of dots (each dot with a linear size of a few nanometers) [3] came both as a surprise and as a challenge. Thus, roughly for the last ten years large efforts have been directed towards harnessing this nanofabrication (nanosculpting [4]) technique. Activities have comprised intensive and fruitful experimental and theoretical work, ranging from basic scientifi questions to the elucidation of a wide range of applications (optoelectronic, magnetic, catalytic, and others). Today, as shown in some of the contributions that follow, a large degree of control has been achieved on the features of the nanopatterns that can be produced experimentally onto a large variety of targets. Nevertheless, many basic questions still remain open on the specifi way in which this type of self-organization process unfolds, whose understanding will undoubtedly impact not only current materials science, but also condensed matter and nonequilibrium physics at large. For this reason surface nanopatterning by ion-beam sputtering (IBS) is still an open, thriving field and we hope that the present special issue conveys a representative view of what has been learnt during this time, and the issues that remain open and drive most current efforts.

The contributions to this special issue are authored by a number of international groups that are leading the systematic study of these topics. They have been grouped roughly into experiments, theory and applications. The experimental contributions firs deal with various morphological aspects of the observed nanopatterns, whether on metals, semiconductors or insulators, at low or at medium ion energies. These are followed by contributions showing the potential of advanced surface characterization techniques, namely x-ray scattering with synchrotron radiation. This technique exploits the benefit of the high-brilliance source, enabling the possibility of in situ and real-time studies. These studies complement the real space and ex situ analysis covered by most other contributions. Then, several papers consider in detail various important issues (like the effect of impurities) whose elucidation is calling for a revision of the physical mechanisms that to date have been considered as the most relevant to control the pattern formation process. After this, some contributions focus on alternative experimental setups (compared to the ones that are most frequently employed, e.g. using single beams of single-charge ions) that can enhance the range of patterns and pattern properties that can be achieved. This opens the way to a set of contributions devoted to applications that explore e.g. magnetic, plastic 
and morphological smoothening properties. This overview on the applications of IBS nanopatterns is placed after the theoretical contributions, and may provide an outlook on the potential implications of progress in the fiel beyond the fundamental aspects of IBS. In spite of the quality of the patterns that can currently be produced, basic issues still remain open on the microscopic mechanisms that control the dynamics. Settling these issues currently requires full synergy between theory and experiments. In this direction, the second large set of papers of this special issue concerns studies that are more theoretically oriented. The firs among these focus on more 'atomistic' or discrete models, some of them covering extensive comparisons with experimental data and/or alternative descriptions, such as those provided by continuum models. The last group of theoretical contributions is devoted to the latter. Here, particular attention is paid (naturally, with a strong correlation to the previous experimental contributions) to the most recent attempts at improving our theoretical understanding over the already classic description by Bradley and Harper [5] of the morphological effects of ion sputtering. This kind of approach is based on the linear cascade and Gaussian approximation developed by Sigmund [6] to describe ion-atom collisions inside the target.

Overall, the scope of the contributions in this issue includes review-type aspects that allow for an update over previously available overviews [7-10]. But we would like to highlight the abundance of novel, original results that faithfully reflec recent substantial progress in this interesting subject. In addition, the papers contain insightful views on how the fiel has evolved in recent years, its impact in other areas, and what can be foreseen in the future.

To conclude, we would like to thank all of the authors for their contributions, and the scientifi editors and technical staff of the journal for their assistance in making this special issue a success.

\section{References}

[1] Cunningham R L, Haymann P, Lecomte C, Moore W J and Trillat J J 1960 Etching of surfaces with 8-keV argon ions J. Appl. Phys. 31839

[2] Navez M, Sella C and Chaperot D 1962 Étude de l'attaque du verre par bombardement ionique C. R. Acad. Sci. Paris 254240

[3] Facsko S, Dekorsy T, Koerdt C, Trappe C, Kurz H, Vogt A and Hartnagel H L 1999 Formation of ordered nanoscale semiconductor dots by ion sputtering Science 2851551

[4] Li J, Stein D, McMullan C, Branton D, Aziz M J, Golovchenko J A 2001 Ion-beam sculpting at nanometre length scales Nature $\mathbf{4 1 2} 166$

[5] Bradley R M and Harper J M E 1988 Theory of ripple topography induced by ion bombardment J. Vac. Sci. Technol. A 62390

[6] Sigmund P 1969 Theory of sputtering. I. Sputtering yield of amorphous and polycrystalline targets Phys. Rev. 184383

[7] Carter G 2001 The physics and applications of ion beam erosion J. Phys D: Appl. Phys. 34 R1

[8] Valbusa U, Boragno C and de Mongeot F B 2002 Nanostructuring surfaces by ion sputtering J. Phys.: Condens. Matter 148153

[9] Chan W L and Chason E 2007 Making waves: kinetic processes controlling surface evolution during low energy ion sputtering J. Appl. Phys. 101121301

[10] Muñoz-García J, Vázquez L, Cuerno R, Sánchez-García J A, Castro M and Gago R 2009 Self-organized surface nanopatterning by ion beam sputtering Towards Functional Nanomaterials ed Z M Wang (New York: Springer) 